\title{
Parabolized stability equation models in turbulent supersonic jets
}

\author{
Daniel Rodríguez ${ }^{1,2 *}, \quad$ Aniruddha Sinha ${ }^{1 \dagger}, \quad$ Guillaume A. Brès ${ }^{3 \ddagger}$ and Tim Colonius ${ }^{1 \S}$ \\ ${ }^{1}$ California Institute of Technology, Pasadena, CA \\ ${ }^{2}$ Universidad Politécnica de Madrid, Spain \\ ${ }^{3}$ Cascade Technologies Inc. Palo Alto, CA
}

The peak noise radiation in the aft direction of high-speed, turbulent jets has been linked to the dynamics of the large-scale structures. We use the parabolized stability equations (PSE) to model these structures as wavepackets associated with instability of the turbulent mean flow. Our past work has demonstrated the utility of these models for subsonic jets; in the present work we extend these methods to supersonic jets. A large eddy simulation database corresponding to an unheated, ideally-expanded Mach 1.5 jet with Reynolds number of 300,000 is employed to extract the necessary input for the PSE (the mean flow and initial conditions) and also to perform comparisons and validations of the computed wavepackets.

By contrast with subsonic jets, when the jet exit velocity is supersonic with respect to the ambient speed of sound, linear stability theory predicts that multiple instability modes, related to resonance of pressure waves within the potential core, can be present in addition to the inflectional instability. ${ }^{1}$ The possible coexistence of different instability mechanisms, the determination of adequate inlet conditions, and their effect on the wavepackets computed are investigated here.

We compare the wavepackets predicted by PSE with fluctuations extracted from the LES data. When performing comparisons, filtering techniques need to be employed in order to extract the coherent, low frequency structures associated with wavepackets. Largescale fluctuations educed using cross-correlation techniques, such as the proper orthogonal decomposition, are shown to compare reasonably well with the PSE wavepackets, but by contrast with subsonic jets, it appears that several POD modes are required to represent the PSE-predicted wavepacket.

\section{Introduction}

The noise generated by a turbulent jet and its subsequent radiation to the far-field is a technological problem of great importance that has received continuous attention for decades. Investigation of the associated flow physics numerically, using direct numerical simulations or large eddy simulations at Reynolds and Mach numbers of practical application remains challenging and computationally expensive even with state-of-the-art algorithms ${ }^{2}$ and supercomputers. An intermediate approach is born from the observation ${ }^{3}$ that the highly-directional peak noise radiated in the aft direction of turbulent jets is associated with the dynamics of the large-scale coherent structures. Relatively coarse descriptions of the non-compact pressure fluctuations, or wavepackets, associated with the large-scale structures can be combined with acoustic projection methods.

Statistical descriptions of the coherent structures observed in forced jets are reminiscent of instability waves, suggesting their modeling as instability wavepackets of the mean turbulent flow. ${ }^{4}$ The conceptual

\footnotetext{
* Marie Curie COFUND fellow, California Institute of Technology, CA, USA and Universidad Politécnica de Madrid, Spain. AIAA Member.

${ }^{\dagger}$ Postdoctoral Scholar, Department of Mechanical Engineering, California Institute of Technology, USA. AIAA Member.

${ }^{\ddagger}$ Senior Research Scientist, Cascade Technologies Inc. AIAA Member.

$\S$ Professor, Department of Mechanical Engineering, California Institute of Technology, USA, AIAA Associate Fellow.
} 
separation of turbulent mixing flow into a mean flow with coherent large-scale and uncoherent small-scale fluctuations (referred to as triple decomposition), and the subsequent modeling of the coherent part as flow instabilities of the turbulent mean flow was formalized by Hussain \& Reynolds. ${ }^{5,6}$ Experimental observations of forced turbulent plane mixing-layers ${ }^{7,8}$ showed that quasi-parallel inviscid linear stability theory delivers good predictions of the statistical large-scale structures for a finite spatial region downstream of the splitter plate, but the agreement is lost as the fluctuation amplitude increases due to strong non-linear interactions between the coherent, uncoherent and mean flow components of the flow. The spatial extent of the region of linear behavior was strongly dependent on the forcing amplitude. Following similar considerations, Mankbadi $\& \mathrm{Liu}^{9}$ pointed out that the evolution of the low-frequency, large-scale statistical structures in turbulent jets is mainly governed by the interaction between the mean flow and the particular structure, with no remarkable interaction between different frequencies. The absence of nonlinear interactions between coherent structures was justified by the relatively small energy content in the individual coherent structures, compared to the energy in the mean flow and in the uncoherent or random fluctuations. This approach was employed in the study of forced supersonic jets, ${ }^{4,9-11}$ for which the measured near field fluctuations were found to be in good agreement with the predictions of linear stability theory. ${ }^{12}$

In the case of natural jets, this approach has only recently begun to deliver satisfactory quantitative predictions. Suzuki \& Colonius ${ }^{13}$ showed that the pressure fluctuations measured just outside of the jet shear layer were consistent with the evolution of linear instability waves computed using locally parallel flow analysis of the jet mean flow. One of the difficulties associated with the experimental observation of the coherent structures in natural jets is the lack of a phase reference, that is trivially determined in forced jets. To overcome this difficulty, Suzuki \& Colonius $^{13}$ considered a series of measurements performed at the NASA Glenn SHJAR facility, in which a microphone phased-array was placed just outside of the turbulent mixing-layer, in a region where hydrodynamic fluctuations are expected to behave linearly and acoustic contamination to be minimal. The careful location of the array and the high-quality, phased measurements performed were instrumental in the successful comparison between theoretical wavepackets and experimental coherent structures. In subsequent work, it was found ${ }^{14}$ that as the Mach number or the jet temperature were increased, the comparison between the linear instability wave predictions and the measurements became poorer. The magnitude of the fluctuations associated with acoustic radiation strongly depends on these two factors (jet exit temperature and Mach number), resulting into a contamination of the measured pressure signal. Proper orthogonal decomposition (POD) of the experimental data was then used in order to filter out the acoustic contamination, and led to outstanding comparisons between linear wavepackets and experimental POD-filtered pressure envelopes and phases for subsonic cold jets, and reasonably good comparisons for heated jets.

A step beyond parallel-flow linear stability theory in the modeling of the wavepackets is achieved with the introduction of the parabolized stability equations (PSE). ${ }^{15-17}$ In our previous works, ${ }^{14,18}$ we employed parabolized stability equations to take into account the mild variation of the jet mean flow along the axial direction and the nonlinear interactions between the different frequency and azimuthal wavenumbers. In these computations, the use of the experimental turbulent mean flow as base flow in the PSE computations partially accounted for the nonlinear interactions present in the flow, and in particular for the Reynolds stresses due to the random, small-scale fluctuations on the establishment of a mean flow, as theoretized by Mankbadi \& Liu. ${ }^{9}$ This approach has been already employed in the literature both in its linear ${ }^{19}$ and nonlinear ${ }^{20}$ versions with promising results. Alternatively, global instability analysis could also be used to provide the description of the linear wavepackets,${ }^{21}$ but at a much higher computational expense.

A fundamental aspect in the wavepacket computation using PSE is the determination of adequate inlet conditions. Besides broader-scoped concerns on the inlet conditions stated in the literature, ${ }^{22,23}$ the PSE integration requires of representative fluctuation shapes, amplitudes and phases for the frequencies and azimuthal wavenumbers of interest. Linear instability theory (LST) for parallel flows shows that subsonic jets can only sustain the Kelvin-Helmholtz instability, either in the form of thin shear-layer instability or as jetcolumn instability. It suffices then to impose the respective Kelvin-Helmholtz eigenfunction as inlet condition for the PSE. However, supersonic jets can sustain additional kinds of instabilities related to the resonance of pressure waves within the potential core. ${ }^{1}$ The sole use of the Kelvin-Helmholtz eigenfunction may not be justified in this case, and more complex inlet conditions may be necessary. If experimental or high-fidelity simulation data is available, the bi-orthogonal system formed by the direct and adjoint eigenfunctions of the LST can be used in order to project a given fluctuation on the different eigenmodes, ${ }^{24-26}$ thus determining the relative importance of the different instability mechanisms at the inlet. 
In the present paper, the PSE model is applied to an isothermal ideally-expanded supersonic turbulent jet, with jet Mach number of 1.5 and Reynolds number based on the nozzle diameter and jet exit velocity of 300,000. A large eddy simulation (LES) database of this configuration, computed using Cascade Technologies flow solver "Charles", ${ }^{2,27,28}$ was used in order to extract the necessary input for the computation of PSE wavepacket models (i.e. the mean flow and initial conditions) and to perform a detailed evaluation of the modeling strategy, as well as to study the physics of the flow that are implicated in the noise generation and radiation. In this respect, standard power spectral density methods were used to extract fluctuation modes in Fourier-Fourier space for time and azimuthal wavenumber. Furthermore, a refined POD technique is also used to extract the large-scale structures more relevant to the noise peak radiation. The remainder of the paper is organized as follows. The flow configuration and LES database are described briefly in section II, while the POD technique employed in the wavepacket extraction is presented in III. Section IV discusses the PSE formulation used. The determination of adequate inlet conditions for the PSE wavepackets is discussed in section V. The PSE wavepackets and their relation with the coherent structures extracted from the LES are discussed in section VI. Finally, some conclusions and possibilities for future directions are exposed in section VII.

\section{Description of the Large Eddy Simulation database}

Large eddy simulation data corresponding to a cold $M_{j}=1.5$ jet are used in the present work with the twofold objective of calibrating the PSE models with the determination of the turbulent mean flow and adequate inlet conditions and validating them by comparing with the wavepackets in the simulation. The LES computations were performed using Cascade Technologies flow solver "Charles". ${ }^{2,27,28}$ The simulation considered the isothermal $\left(T_{j}=T_{\infty}\right)$ ideally-expanded jet flow emanating from a convergent-divergent roundnozzle at Reynolds number $R e=300,000$.

In what follows, non-dimensional form of the physical quantities will be used except where stated otherwise. The reference scale is the jet diameter $D$, the reference velocity is the ambient speed of sound $c_{\infty}$, yielding the reference time $t_{r e f}=D / c_{\infty}$. Pressure is made dimensionless with the physical dynamic pressure $\rho_{\infty} c_{\infty}^{2}$, and density with the far-field density $\rho_{\infty}$. Besides this non-dimensionalization, some quantities are defined with variables different from the reference ones: the Reynolds number is based on the jet exit conditions: $R e=\rho_{j} U_{j} D / \mu_{j}$, that is related to the acoustic Reynolds number $R e_{a}=\rho_{\infty} c_{\infty} D / \mu_{\infty}$ by $R e=R e_{a} M_{j}$. The dimensionless frequency used to show results will be the Strouhal number defined as $S t=f D / U_{j}$ instead of the Helmholtz number $H e=f D / c_{\infty}$ that would be consistent with the dimensionless form employed. This dimensionless form is common to the LES database and the formulation of the parabolized equations.

Time-averaged quantities such as the mean flow variables or the averaged Reynolds stresses were computed using the total simulation time (after initial transients) of $t_{\text {tot }} \approx 210 D / c_{\infty}$, considered to be long enough to have statistical convergence of the stationary quantities. The simulation was performed on an unstructured mesh with $43 \times 10^{6}$ control volumes and the total size of the transient LES database is approximately $18 \mathrm{~Tb}$. In order to calibrate and validate the PSE wavepacket models, the time-dependent flow field variables were first undersampled in spatial and temporal resolutions in order to deliver a reasonable-sized dataset. Data were interpolated to an uniform structured cylindrical mesh with $N_{x} \times N_{r} \times N_{\theta}=41 \times 400 \times 72$ points, resulting in spatial spacings $\Delta x=0.5 D$ and $\Delta r=0.0125$, and a constant time step $\Delta t=0.02 D / c_{\infty}$. In addition, the data were interpolated to a virtual cone akin to the microphone array used in UTRC experiments. The time series are double Fourier transformed in the frequency domain and in the azimuthal direction. The corresponding amplitudes are computed using standard sampling techniques as power spectral densities (PSD), and then square-rooted. The accuracy of this process depends on the quality of the used data, i.e. resolution in the azimuthal direction $N_{\theta}$ and time step $\Delta t$ and total number of time steps $N_{s}$. Proper orthogonal decomposition will also be used, the quality of which will also depend on the data quality. Some studies were conducted in order to determine the necessary resolution in time and space for the convergence of the fluctuation PSDs. These studies, not reported here, showed that using the total simulation time delivers converged statistics. The spatial resolution in the axial and radial directions cannot be further reduced, otherwise important details in the lip-line region, where fluctuations are stronger, would not be adequately described. The resolution on the azimuthal direction can be reduced to $N_{\theta}=24$ equally spaced points and still delivers converged results for the azimuthal modes of interest. Finally, for the range of frequencies of interest here, the time step in the temporal Fourier transforms can be increased to $\Delta t=0.04 D / c_{\infty}$, implying a two-fold reduction in memory and computing time. 


\section{Extraction of wavepackets from LES data using Proper Orthogonal Decomposition}

The PSE models are intended to capture the coherent wavepacket motions at low frequency. In order to validate these models with LES data, which resolves motions over a broad range of length scales, these structures must be educed with appropriate statistical techniques. The power spectral density (PSD) is partially effective, isolating motions at low frequencies. But, as we show below, techniques based on cross correlations are needed to educe the coherent structure. In this study, the proper orthogonal decomposition $(\mathrm{POD})^{29}$ is employed to extract the structures with correlation over a significant spatial region. Such an approach has been previously used to data from low Reynolds number turbulent jets ${ }^{28}$ and with microphone array data ${ }^{14}$ from subsonic jets.

The LES flow field is considered to be statistically stationary in time and homogeneous along the azimuthal direction, and thus Fourier modes in frequency and azimuth are introduced, in the same manner as is done in the derivation of the parabolized stability equations:

$$
\tilde{q}^{k}(x, r ; m, n)=\int_{t=0}^{2 \pi / \omega} \int_{\theta=-\pi}^{\pi} q(x, r, \theta, t) \exp [\mathrm{i}(m \theta-n \omega t)] d t d \theta .
$$

The index $k$ denotes the time segment used in the temporal Fourier transform.

The use of different norms in the computation of POD modes of a turbulent jet was first done by Freund \& Colonius, ${ }^{30}$ showing that the resulting decomposition may be highly dependent on the physical variables of interest. The flow variable of interest here is the fluctuating pressure field, and the POD should be geared toward extracting its dominant features. Thus, the inner product is defined as

$$
<\tilde{q}^{i}, \tilde{q}^{k}>=\int_{x} \int_{r}\left(\tilde{p}^{i}\right)^{\dagger} \tilde{p}^{k} r d r d x
$$

with $\dagger$ denoting complex conjugate.

The frequency-domain variant of Sirovich's snapshot POD method ${ }^{31}$ is employed. A segment-to-segment cross-correlation matrix is defined now by applying the inner product to pairs of Fourier transformed perturbation fields, corresponding to different segments of data:

$$
\tilde{S}_{i j}=<\tilde{q}^{i}, \tilde{q}^{j}>
$$

POD modes are the optimal basis of orthogonal functions that minimizes the square mean error between the fluctuations $\tilde{q}^{k}$ and its projection on a basis of orthogonal functions. The $l^{\text {th }}$ POD mode to be computed can be written as a linear combination of the different transformed segments:

$$
\tilde{\phi}^{l}(x, r ; m, n)=\sum_{k} \beta_{k}^{l}(m, n) \tilde{q}^{k}(x, r ; m, n),
$$

where the coefficients $\beta_{k}^{l}$ are obtained as the eigenvectors of the eigenvalue problem

$$
\tilde{S} \beta^{l}=\lambda^{l} \beta^{l} .
$$

The non-negative eigenvalues $\lambda$ arranged in descending order indicate the order of optimality of the respective POD eigenfunctions. The first POD mode corresponds to the largest $\lambda$, the second corresponds to the second largest, and so on.

The Fourier decomposition in the homogeneous azimuthal direction reduces the dimensionality of the POD problem by one. The POD performed on the entire three-dimensional domain reduces to a twodimensional POD in $x-r$; this will be termed planar-POD henceforth. Alternatively, the LES data on a

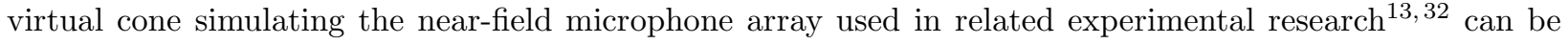
used exclusively. The POD on the virtual cone reduces to a one-dimensional POD along a meridional line; this will be called line-POD.

\section{Parabolized stability equations}

Parabolized stability equations (PSE) ${ }^{15-17}$ represent a generalization of the parallel-flow linear stability theory (LST) for flows with a mild variation in the streamwise direction, also permitting the nonlinear 
interaction of the different modes. The technique was originally devised for studying transition in laminar flows. ${ }^{17}$ Here, PSE is proposed as a technique for reduced-order modeling of the wavepackets linked to instability modes that are implicated in the peak noise radiation to aft angles in turbulent jets.

The total flow field $q$ is decomposed into a mean (time-averaged) and axisymmetric component $\bar{q}$ and its fluctuations $q^{\prime}$. The formulation of the equations used for the PSE considers the velocity components in a cylindrical coordinate system $u_{x}, u_{r}$ and $u_{\theta}$, the dimensionless pressure $p$ and the specific volume $\zeta=1 / \rho$ as the flow variables. Only round-nozzle jets are considered here. The fluctuating part is then written as a sum of Fourier modes in the azimuthal direction and in frequency where $m$ is the azimuthal wavenumber, $\omega$ is the fundamental circular frequency, $n$ is the harmonic number, and $\chi_{m n}$ is the modal function corresponding to the mode $(m, n)$. In the practical solution of nonlinear PSE, the azimuthal and frequency modes must be truncated, involving a finite number $(M, N)$ of modes. The unresolved modes are then formally gathered in the term $q^{\prime \prime}$, and the fluctuation field is written as

$$
q^{\prime}(x, r, \theta, t)=\sum_{n=-N}^{N} \sum_{m=-M}^{M} \chi_{m n}(x, r) \exp (\mathrm{i}(m \theta-n \omega t))+q^{\prime \prime} .
$$

The mean flow is a function of the axial and radial directions $(x, r)$, but a slow variation of its properties along the axial direction is assumed. This assumption permits the decomposition of $\chi_{m n}$ into a slowly varying shape function (that evolves in the same scale as the mean flow) and a rapidly varying wave-like part:

$$
\chi_{m n}(x, r)=A_{m n}(x) \cdot \hat{q}_{m n}(x, r)=\exp \left(\mathrm{i} \int_{x} \alpha_{m n}(\xi) d \xi\right) \cdot \hat{q}_{m n}(x, r) .
$$

Here $\alpha_{m n}(x)$ is a complex axial wavenumber, for which a mild variation is also assumed.

Introducing the decomposition (6-7) into the compressible Navier-Stokes, continuity and energy equations and subtracting the terms corresponding to the mean flow, we arrive at the system of equations

$$
\begin{gathered}
\left(\mathcal{L}^{0}-\mathrm{i} n \omega \mathcal{L}^{t}+\mathcal{L}^{x} \frac{\partial}{\partial x}+\mathcal{L}^{r} \frac{\partial}{\partial r}+\mathrm{i} m \frac{\mathcal{L}^{\theta}}{r}+\right) \hat{q} \\
+\frac{1}{R e_{\infty}}\left(\mathcal{V}^{0}+\mathcal{V}^{x} \frac{\partial}{\partial x}+\mathcal{V}^{r} \frac{\partial}{\partial r}+\mathrm{i} m \frac{\mathcal{V}^{\theta}}{r}+\mathcal{V}^{x x} \frac{\partial^{2}}{\partial x^{2}}+\mathcal{V}^{r r} \frac{\partial^{2}}{\partial r^{2}}\right. \\
\left.-m^{2} \frac{\mathcal{V}^{\theta \theta}}{r^{2}}+\mathcal{V}^{x r} \frac{\partial^{2}}{\partial x \partial r}+\mathrm{i} m \frac{\mathcal{V}^{r \theta}}{r} \frac{\partial}{\partial r}+\mathrm{i} m \frac{\mathcal{V}^{x \theta}}{r} \frac{\partial}{\partial x}\right) \hat{q}=\frac{\hat{F}_{m n}}{A_{m n}}+\frac{F_{m n}^{\prime \prime}}{A_{m n}} .
\end{gathered}
$$

Expressions for the linear operators $\mathcal{L}$ and $\mathcal{V}$ can be found in Appendix A. For brevity, the subscript have been dropped from the shape function and wavenumber in the previous expression. The left-hand-side in (8) is a linear spatial operator for the mode $(m, n)$, while the right-hand-side accounts for all the nonlinear contributions to the modal evolution. Linear PSE are obtained setting the right-hand-side equal to zero. The contributions resulting from interactions of the resolved modes are comprised in the function $\hat{F}_{m n}(x, r)$, while those interactions in which the unresolved modes are involved are gathered in the function $F_{m n}^{\prime \prime}$. In nonlinear computations considering laminar or transitional flows all the dynamically relevant modes are resolved, and $F_{m n}^{\prime \prime}$ is neglected. In the case of the natural turbulent jets of interest here, a broad spectrum exists and the truncation of the modes is always arbitrary. Consequently, the function $F_{m n}^{\prime \prime}$ is not necessarily negligible and may need to be modeled.

Equation (8) requires an additional condition to be closed. Following Herbert, ${ }^{17}$ the normalization condition

$$
\int_{0}^{\infty} \hat{q}^{\dagger} \frac{\partial \hat{q}}{\partial x} r d r=0
$$

is imposed individually to every mode, removing the exponential dependence on the shape function $\hat{q}$. Here the superscript $\dagger$ denotes complex conjugation.

The system of equations is discretized using fourth-order central finite differences in the radial direction, closing the domain with the characteristic boundary conditions of Thompson. ${ }^{33}$ The boundary conditions at the centerline are derived following Mohseni \& Colonius. ${ }^{34}$ The streamwise derivative is approximated using an implicit first-order Euler scheme with a relatively large step size $\Delta x$, in order to prevent ellipticityrelated instabilities ${ }^{35}$ in the marching procedure. For each successive axial location, the system (8) is solved iteratively: the $\alpha$ and $\hat{q}$ converged in the previous location are used as initial guesses. 


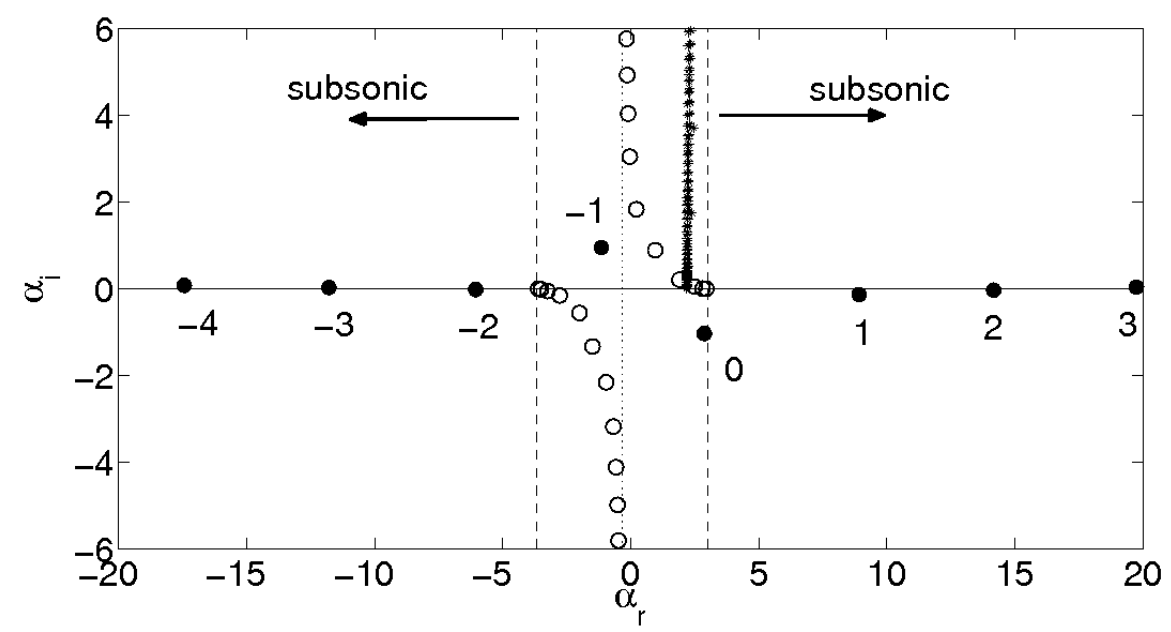

Figure 1. Spatial LST eigenspectrum at $S t=0.35, m=0$ and $x / D=0.5$.

\section{Inlet conditions for PSE wavepackets using LES data}

The PSE solution requires an initial condition (radial variation of the PSE mode) to be specified at the upstream boundary of the domain, typically near the nozzle exit. The Kelvin-Helmholtz mode is the only convective instability in subsonic jets, and so it is expected to be the determinant of the PSE mode shape over any significant axial extent. In the absence of further information, Gudmundsson \& Colonius ${ }^{14}$ initiated the PSE with the Kelvin-Helmholtz mode obtained from the parallel-flow linear stability analysis applied to the turbulent mean flow field at a cross-section near the nozzle exit. The LES database contains full-field time-resolved flow information. This represents an opportunity to revisit the initialization of the PSE computations.

The biorthogonal projection approach was introduced in the field of hydrodynamic instability by Salwen $\&$ Grosch $^{24}$ and in flow control by Hill. ${ }^{25}$ It has been used in the past to filter the fluctuation fields from experiments and numerical simulations. ${ }^{26,36,37}$ In the present work, this method is adopted to determine the initial conditions for the PSE computations that provide the best possible description of the instability wavepackets while filtering out other perturbations present at the initial axial station. Section V.A briefly revisits the linear stability analysis. Section V.B describes the biorthogonal projection technique, and considers its use for filtering the LES data. Section V.C compares the results of linear PSE initiated with different sets of eigenmodes obtained with the above method.

\section{A. Parallel-flow linear stability theory}

The PSE linear operators are used as the departure point in the definition of a local linear stability eigenvalue problem (EVP), thus analyzing the eigenspectrum of the PSE approximation, instead of the usual compressible linearized Navier-Stokes equations for parallel flow. In particular, second derivatives in the axial direction are neglected on account of the high Reynolds number of the jet. As pointed out by Li \& Malik, ${ }^{35}$ neglecting these terms eliminates a continuous branch of stable upstream propagating modes that are of no significance in the downstream evolution of the wavepackets.

After linearization and neglect of non-parallel effects, the discretized matrix formulation of the PSE (8) can be rewritten as the matrix EVP

$$
\mathcal{A} \hat{q}=-\mathrm{i} \alpha \mathcal{B} \hat{q}
$$

for the complex axial wavenumber $\alpha$. The eigenvectors are denoted by $\hat{q}$. The generalized matrix eigenvalue problem (10) is discretized using exactly the same numerics developed for the PSE. The generalized Schur decomposition (QZ algorithm) implemented in the LAPACK routine ZGGEV is then used to compute the full eigenspectrum along with the right and left eigenvectors.

Figure 1 shows a typical eigenspectrum, obtained for $S t=0.35$ and $m=0$ at $x / D=0.5$. Apart from the Kelvin-Helmholtz instability of the shear layer, the spectrum contains families of eigenmodes corresponding 
Table 1. Ratio between peak pressure and axial velocity magnitudes $\left(|\hat{p}|_{\max } /\left|\hat{u}_{x}\right|_{\max }\right)$ of the eigenvectors in figure 2 .

Instability waves - subsonic instability and Kelvin-Helmholtz

\begin{tabular}{cccccc}
\hline$k$ & -2 & -1 & 0 & 1 & 2 \\
$|\hat{p}|_{\max } /\left|\hat{u}_{x}\right|_{\max }$ (direct) & 0.7623 & 0.1588 & 0.0672 & 0.5349 & 1.1293 \\
$|\tilde{p}|_{\max } /\left|\tilde{u}_{x}\right|_{\max }$ (adjoint) & 0.8718 & 0.4669 & 0.0670 & 0.5322 & 0.8167
\end{tabular}
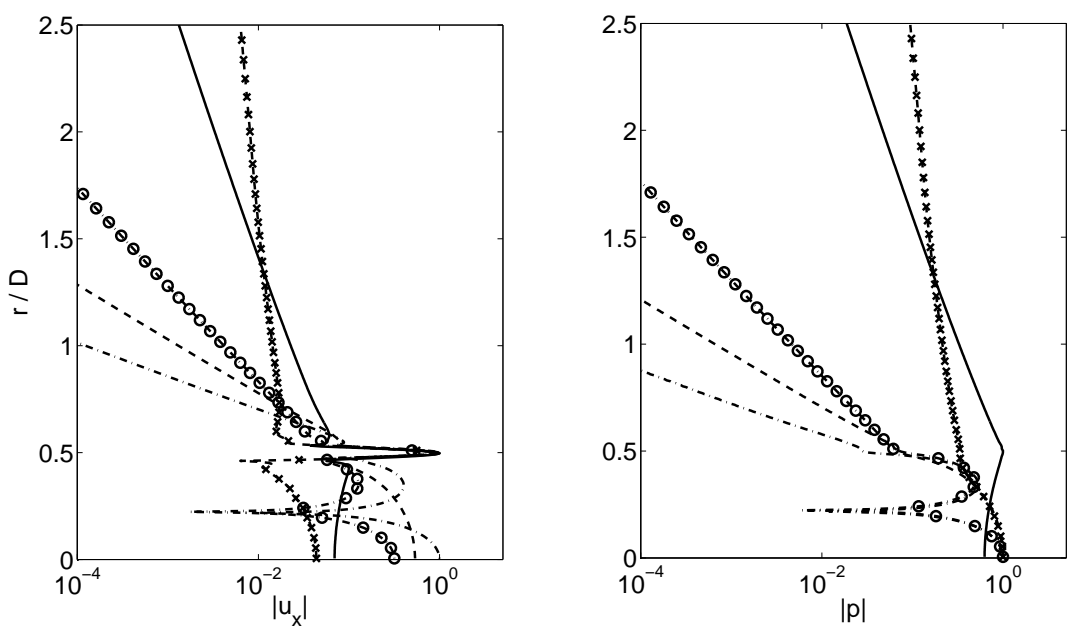

Figure 2. Linear stability eigenfunctions for $S t=0.35, m=0$ and $x / D=0.5$. Axial velocity (a) and pressure (b) components of the eigenvectors $k=-2$ (० dashed-dotted); $k=-1$ ( $\times$ dashed); $k=0$ (solid); $k=1$ (dashed) and $k=2$ (dashed-dotted).

to pressure, vorticity and entropy waves inside of the potential core and in the outer field. Only the family of discrete eigenmodes denoted by filled circles in figure 1 is of interest here. This branch comprises two out of the three different instability mechanisms in supersonic jets, namely Kelvin-Helmholtz and subsonic instability waves. Supersonic instability waves are not possible for this Mach number, as explained by Tam $\& \mathrm{Hu}^{1}$

Only a finite number of spatially amplified modes are present. These eigenmodes can be enumerated according to the number of peaks $k$ that the eigenfunctions present inside the potential core. The first one corresponds to the Kelvin-Helmholtz instability - it is characterized by a marked peak at the lipline $(r / D=0.5)$ and has no peaks inside the core $(k=0)$. Modes $k=1, k=2$ and so on are unstable waves propagating with subsonic phase speed inside the potential core. To the left of mode $k=0$ in the eigenspectrum, the other branch of subsonic waves is found, referred to as $k=-1,-2, \ldots$. The axial velocity and pressure components of the eigenvectors associated with the different modes are shown in figure 2, and the peak pressure to velocity ratio are presented in table 1.

The relative importance of the pressure component is increased with the number of core peaks $k$. The axial velocity profile has a peak in the lip-line region for all the different instability modes. The subsonic modes with the same number of peaks, e.g. $k=1$ and $k=-1$, have peaks and nodes inside the potential core at the same locations, revealing their resonant nature. Given that the centerline peak dominates the pressure component, these eigenfunctions are nearly identical inside the core (figure 2, right). Outside the mixing region, the decay in the radial direction is different for each eigenmode, on account of the differences in the axial wavenumber $\alpha_{r}$. In this region the base flow is approximately uniform, and linear theory predicts a decay rate $\sim r^{-1 / 2} \exp \left(-r \sqrt{\alpha_{r}^{2}-\omega^{2}}\right)$, that is visible in the eigenfunctions (figure 2). The eigenmode $k=-1$ with the smallest wavenumber decays slower than the others, and at first, this may imply its relevance to the noise radiated to the far field. However, as a counterpoint, this wave is also quickly damped in the axial direction, making such simplistic deductions difficult. 

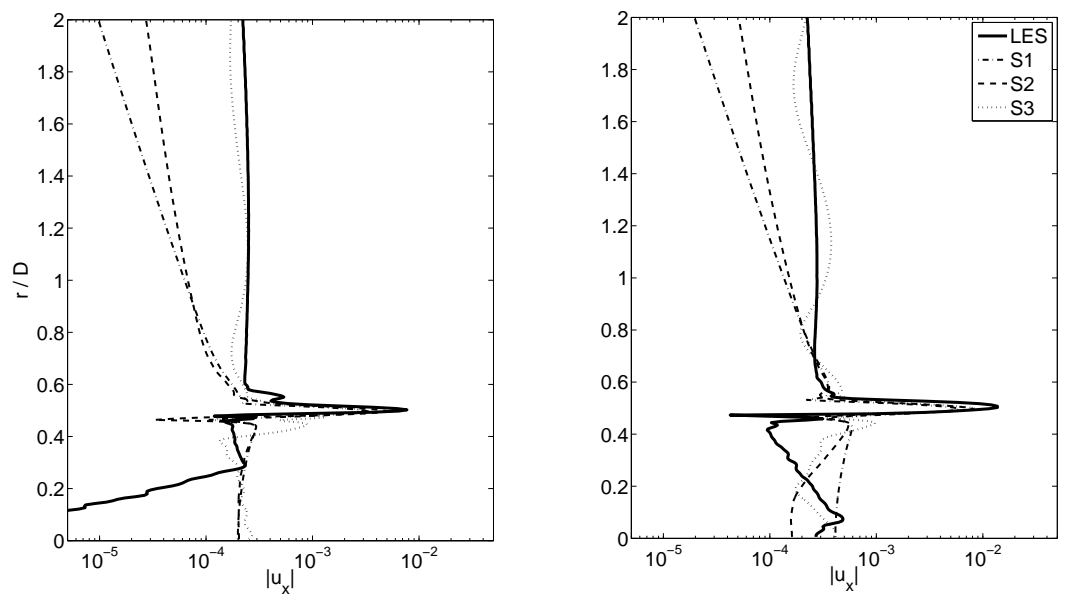

Figure 3. Fluctuation axial velocity component at $x=0.25$ (left) and $x=0.5$ (right), extracted from LES (thick black solid), and as the projection on different subsets of linear instability eigenmodes: $S 1$ (dashed-dotted), $S 2$ (dashed), $S 3$ (dotted). Strouhal and azimuthal wavenumbers are $S t=0.35$ and $m=0$.

\section{B. Biorthogonal projection}

The linear operators describing the eigenvalue problem (10) are non-orthogonal, a property inherited from the linearized Navier-Stokes equations, and consequently the associated eigenvectors $\hat{q}$ are not orthogonal. The adjoint problem is introduced in order to construct the following bi-orthogonality relation between the direct and adjoint eigenvectors:

$$
\left(\alpha_{k}-\alpha_{l}\right) \tilde{q}_{k}^{\dagger} \mathcal{B} \hat{q}_{l}=0 \quad \Rightarrow \quad \tilde{q}_{k}^{\dagger} \mathcal{B} \hat{q}_{l}=C_{l} \delta_{k l},
$$

where $\tilde{q}_{k}$ is the adjoint eigenfunction corresponding to the eigenvalue $\alpha_{k}, \delta_{l k}$ is a Kronecker delta function, and $C_{l}=\tilde{q}_{l}^{\dagger} \mathcal{B} \hat{q}_{l}$.

A given perturbation field $q^{\prime}$, previously Fourier transformed in time and azimuthal direction to $(S t, m)$, can be written as the weighted sum of the desired eigenmodes:

$$
q^{\prime}=\sum_{k} a_{k} \hat{q}_{k}+q_{\text {out }}, \quad a_{k}=\left(\tilde{q}_{k}^{\dagger} \mathcal{B} q^{\prime}\right) /\left(\tilde{q}_{k}^{\dagger} \mathcal{B} \hat{q}_{k}\right)
$$

The component $q_{\text {out }}$ represents the fluctuations irrelevant to the wavepacket modeling; they are to be filtered out based on heuristics elaborated below. The radial shape of the adjoint eigenfunctions determines the regions that have the largest contributions to the amplitude weights $a_{k}$ of each eigenmode. Most of the adjoint eigenfunctions (not shown) have a peak in the vicinity of the inflection point. The implication is if the arbitrary perturbation field $q^{\prime}$ contains large amplitude fluctuations at the inflection point, then large values of weights are to be expected.

Following this procedure, initial conditions for the PSE can be constructed as a linear combination of a chosen subset of the LST eigenmodes, with the relative amplitudes obtained from the numerical simulation data. The PSE modal function at the inlet location $x=x_{\text {in }}$ corresponding to equation (7) is then written as

$$
\chi_{m n}\left(x_{\mathrm{in}}, r\right)=\sum_{k} a_{m n, k} \cdot \hat{q}_{m n, k}(r) .
$$

Figure 3 displays the fluctuation profiles of axial velocity in the $S t=0.35, m=0$ mode at $x=0.25$ and $x=0.5$. A comparison is made between the profiles extracted directly from the LES and their projection on different subsets of eigenmodes. Three sets of eigenmodes are considered in the projection. The first subset $S 1$ corresponds to the Kelvin-Helmholtz instability $(k=0)$ alone. The second subset $S 2$ comprises $S 1$ and the nearer subsonic instability eigenmodes $(S 2 \ni k=\{-1,0,1\})$. Subset $S 3$ includes the Kelvin-Helmholtz and the first 8 subsonic instability waves $(S 3 \ni k=\{-4,-3, \cdots, 3,4\})$ in addition of 40 other eigenmodes in the eigenspectrum: 10 downstream moving pressure waves, 10 upstream moving pressure waves, 10 core vorticity 
modes and 10 entropy vorticity modes. A finite and rather small number of eigenmodes are considered, even in the subset $S 3$, and thus fine structures cannot be captured, especially inside the potential core and in the outer region, where respectively infinite discrete and continuous spectra exist. Due to the non-normality of the eigenmodes, the projection on the eigenmode subsets is not necessarily bounded by the LES profile, and consequently the convergence of the projection as the number of eigenmodes is increased is not monotonous.

The thick black curve in figure 3 (left) corresponding to the LES data at $x=0.25$ decays inside the potential core since the LES does not impose any perturbations at the nozzle. As the turbulent mixing layer develops in the axial direction, the disturbances spread towards the jet axis, as can be seen in figure 3 (right). Adequate recovery of this behavior is not attained in the projection due to the relatively small number of core modes retained. On the other hand, in the mixing region surrounding the inflection point $(r=0.5)$ the sharp peak of the LES profile is accurately described even by the projection on the Kelvin-Helmholtz eigenmode alone $(S 1)$. This indicates that the disturbance field is dominated in this axial region by the inflectional instability. The peak of the axial velocity magnitude is at least one order of magnitude higher than the perturbations at any other radial location, and dominates over the other velocity components. However, the behavior of the Kelvin-Helmholtz shape functions diverges from the LES profile as $r \rightarrow \infty$. The addition of the subsonic instability waves improves the agreement in the outer region slightly.

In any case, all the instability waves decay exponentially in the radial direction, and cannot explain the trend observed in the LES data. When the outer flow pressure waves are introduced in the projection (S3), the LES trend is recovered.

\section{Sensitivity of linear PSE results to the initial conditions}

The foregoing discussion has revealed that the linear combination of the Kelvin-Helmholtz eigenmode and the first two subsonic instability modes comprise most of the LES fluctuations in the shear-layer region. However, the Kelvin-Helmholtz eigenmode exhibits much stronger spatial amplification in the downstream direction, so that the relevance of the other two eigenmodes in the downstream evolution of the PSE modes is unclear.

Figure 4 compares the solution of the linear PSE initialized with different eigenmode projections with the solution obtained by imposing the LES disturbance profile directly as the inlet condition. The two eigenmode subsets $S 1$ and $S 2$, and the initial axial locations $x / D=0.25,0.5$ and 1 are considered in the comparison. The cross-section at which the PSE integration starts is indicated in the figures.

Small differences are observed in the vicinity of the initial axial location that decay fast as the integration evolves downstream. Downstream of the $x / D=2$ section, the fields corresponding to $S 1$ and $S 2$ are visually indistinguishable. The main difference between the PSE solution initialized with the LES profile and those with biorthogonal projections concerns the regions far outside the mixing layer, and more precisely in the manner in which the perturbations decay towards the far-field. This disagreement is expected on account of the projections shown in figure 3. It was surmised that the inclusion of the $k=-1$ mode may be important for accuracy in the outer region. However, its highly damped character is evidently precluding this effect.

It has been discussed earlier that modeling the wavepackets in the near field immediately outside the shear layer is the most pertinent for predicting peak aft angle noise radiation. In this regard, the above analysis indicates that the predictions in this critical region are quite insensitive to the various initial conditions considered, as far as the contribution of the Kelvin-Helmholtz eigenmode is included. There are two main reasons for this. First, the Kelvin-Helmholtz instability is concluded to be the leading mechanism in this particular jet configuration (which is not necessarily true for all jets). Second, the PSE integration accounts for a single axial wavenumber $\alpha$ for each $(S t, m)$ mode, and converges to the most unstable mode (KelvinHelmholtz instability) thus damping the other contributions. In the remainder of this work, only the KelvinHelmholtz mode from the parallel-flow LST is used to determine the inlet conditions for the PSE integration.

\section{PSE wavepacket models compared with POD modes}

\section{A. Linear PSE compared with planar-POD}

This section discusses a proper orthogonal decomposition of the LES data on the full computational domain, considering the two inhomogeneous spatial directions $(x$ and $r$ ) and Fourier modes in the homogeneous azimuthal direction and stationary time direction. In the computation of these POD modes, all the relevant spatial locations within the jet flow are correlated at the same time through the inner product (2), extracting 

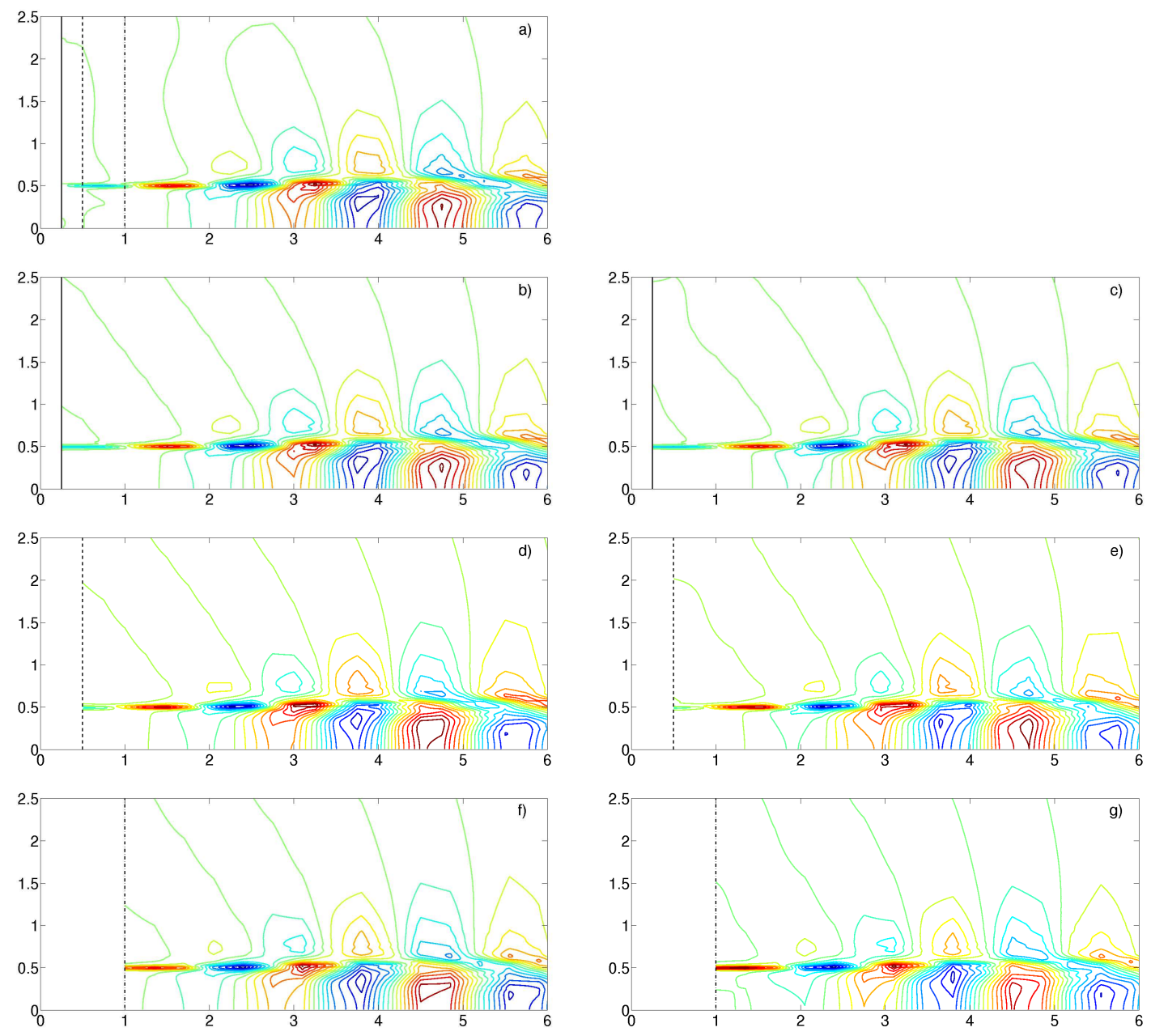

Figure 4. Disturbance axial velocity component obtained as the solution of the linear PSE, at $S t=0.35, m=0$. The initial axial location for each case is $x=0.25(\mathbf{a}, \mathbf{b}, \mathbf{c}), x=0.5(\mathbf{d}, \mathbf{e})$ and $x=1(\mathbf{f}, \mathbf{g})$, as denoted by the vertical lines in the different subfigures. The LES data is used directly as initial condition in case (a). Cases (b), (d) and (f) correspond to the projection using the eigenmodes subset $\mathrm{S} 1$, and cases (c), (e) and (g) correspond to the subset S2.

flow structures that are coherent over significant distances. One potential benefit of computing POD modes of the whole domain is the possibility of performing direct comparisons of the PSE modes with the extracted structures, without resorting to interpolations localized in particular cross-sections or conical surfaces (as $\mathrm{in}^{14}$ ) that can be chosen adequately in order to deliver optimal comparisons. For brevity, the presentation will consist of results for three different frequencies $S t=\{0.1,0.3,0.5\}$ and $m=0$ and 1 azimuthal modes, which cover the range of large-scale structures most significant for the aft peak noise radiation.

Figure 5 depicts the POD eigenspectra for the low-order Fourier modes. It is readily apparent that the POD is unable to reveal a single dominant coherent mode in any of the Fourier modes, that may have been directly comparable with PSE predictions. The relative flatness of the spectra means that many mutually orthogonal modes are dynamically significant in the pressure field. It is also noticed that the flatness of the spectra increases at higher frequencies and azimuthal numbers.

Figures 7-9 show comparisons of the linear PSE results with the first two POD eigenfunctions for the Fourier modes under consideration. The linear PSE modes have arbitrary absolute amplitude and phase, so that only their relative amplitudes and phase are pertinent in the figures. The POD yields an orthogonal basis set, so that the PSE solution can be orthogonally projected onto the individual POD modes using the inner product defined previously. The magnitude of the projection coefficient is a measure of the correlation 

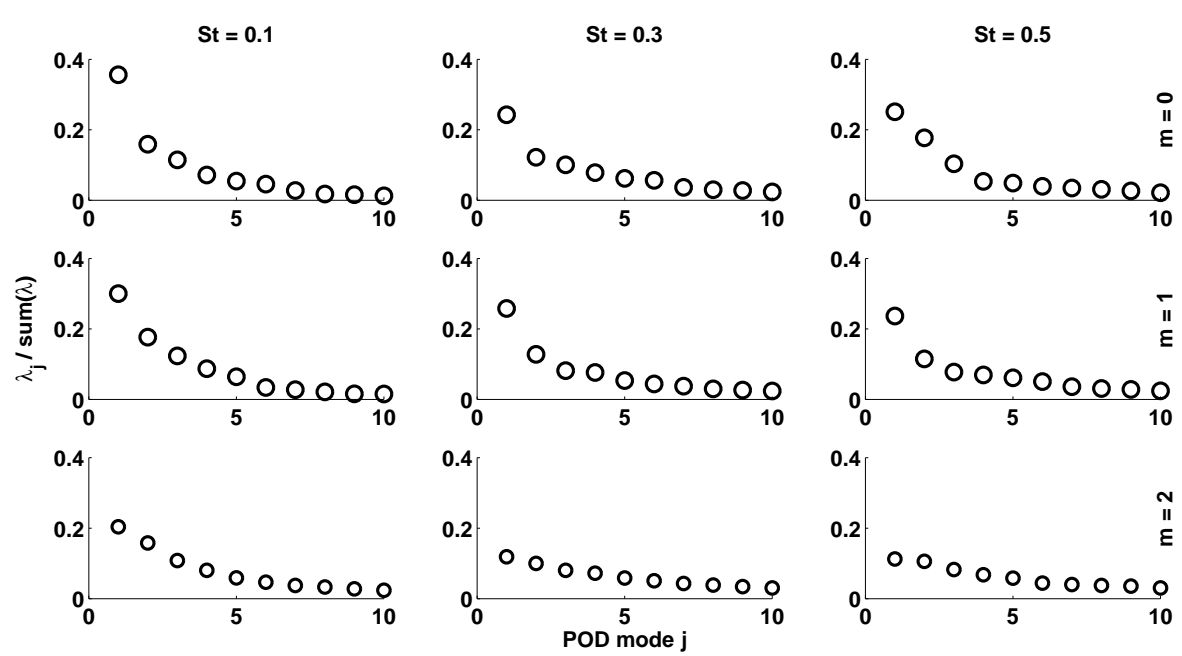

Figure 5. Eigenspectra of planar-POD for different $S t$ and $m$.

of the PSE solution $\chi(x, r)$ (equation 7 ) with the corresponding POD mode $\tilde{\phi}^{j}(x, r)$ (equation 4):

$$
\beta_{j}=\frac{<\chi(x, r), \tilde{\phi}^{j}(x, r)>}{\|\chi(x, r)\|\left\|\tilde{\phi}^{j}(x, r)\right\|} .
$$

Figure 6 depicts the squared projection coefficients. Note that due to the orthogonality property of the POD modes, the sum of the squared $\beta_{j}$ coefficients must be equal to unity. For most cases, a small number of POD modes is required in order to reconstruct the PSE modes for the lowest frequencies, so that the sum of the first $4 \beta_{j}^{2}$ coefficients amounts to around $80 \%$ of the total. The agreement deteriorates as the frequency increases over $S t=0.3$, but it is presently unclear if the disagreement is motivated by physical reasons or due to a poorly resolved quadrature: in order to expedite the post-processing, the LES data was extracted on a coarsened axial grid with uniform spacing of $\Delta x=0.5 D$ which is perhaps too coarse for the smallest wavelengths associated with the higher frequencies considered herein.

The results for $S t=0.1, m=0$, on figure 6 indicate that the first two POD modes together reconstruct the majority of the corresponding PSE solution. Figure 7 demonstrates the visual resemblance. The close connection between POD modes and Fourier modes is extensively described in the literature. ${ }^{29}$ In particular, considering POD of an arbitrary wavepacket, rapid variations in the wavepacket's amplitude envelope or phase speed would require more POD modes in the reconstruction. At lower frequencies, the PSE modes here represent wavepackets with more gradual variations. This may explain the effectiveness of the POD filtering in these cases. The modeling assumptions that go into PSE (specifically, the forced removal of upstream propagating waves) render it a relatively ineffective predictor of the acoustic signature in the near field. This is seen in the departure of the pressure contours outside the virtual cone from those captured by the POD modes. The foregoing discussions also apply to the $S t=0.3$ modes. The $m=1$ case is efficiently reconstructed using the first few POD modes (see figure 6), and the same conclusion can be drawn from figure 8. The $m=0$ case is different in that, the POD modes display strong acoustic fields resembling Mach wave radiation patterns. The $S t=0.3, m=0$ mode is indeed an efficient radiator. The PSE mode displays a much weaker acoustic field for reasons stated above, and thus requires several POD modes for its reconstruction. Clearly, full-field planar-POD is not the optimal filter for comparison here. The previous discussion of figure 6 vis-a-vis the $S t=0.5$ modes has anticipated the issue seen in figure 9 . Namely, the PSE solution represents a wavepacket with wavelength close to the grid resolution of the extracted LES data. The POD modes are displaying wavepackets with much larger wavelengths, most probably due to aliasing. This case will not be discussed further.

In summary, PSE appears to be effectively predicting the wavepacket signatures that dominate the hydrodynamic near field. The comparison with POD-filtered data is more challenging than in the subsonic jets, as the increased importance of the acoustic field in the total fluctuations as Mach number increases may require several POD modes to mutually cancel in order to yield the hydrodynamic field obtained in PSE. 


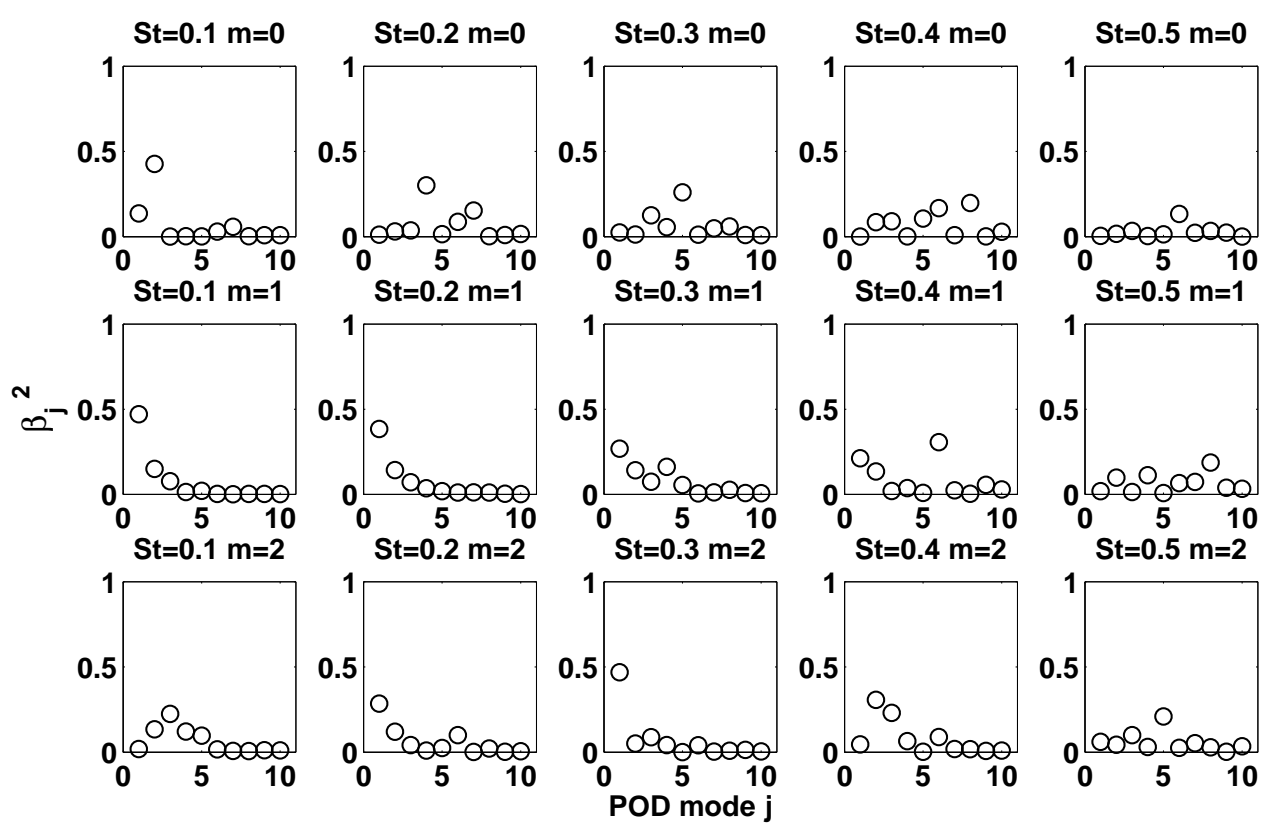

Figure 6. Squared magnitudes of projection coefficients $\beta_{j}$ for the respective PSE solutions on to the planarPOD modes.

However, careful analysis of the planar-POD results presented herein reveals acceptable fidelity of the PSE model.

\section{B. Linear PSE compared with line-POD on virtual cone}

The results in the preceding section exhibit a remarkable qualitative agreement between the PSE modes and the structures associated with the first planar-POD modes for a given $S t$ and $m$ numbers. However, making quantitative comparisons using the full domain structures results troublesome due, in part, to the coarse resolution in the axial direction of the extracted LES data, but also to the different physical mechanisms that are recovered together in the same POD modes. These mechanisms apparently include the linear hydrodynamic wavepackets to be compared with the PSE modes, but also acoustic radiation observable towards the far-field and the possibility of important non-linear interactions in the turbulent mixing region and inside of the potential core. Our earlier work on validation of PSE models ${ }^{14}$ focused on comparisons with experimental measurements made at NASA SHJAR on a phased microphone conical cage. ${ }^{13}$ This array was designed so as to resolve the signature of wavepackets in the hydrodynamic pressure field for the energetically-relevant frequency and azimuthal modes, and allows a compact quantitative evaluation of PSE models. Analogous comparison is done here by extracting the pressure data on an similar virtual cone, the exact location of which corresponds to the rotating cage array that was specifically designed at UTRC to perform measurements on supersonic round jet at the same conditions monitored here. ${ }^{32}$ The virtual microphones are located on a cone of $7^{\circ}$ half-angle between $x / D=3$ and 20 , with the most upstream sensor being at $r / D=1.25$. The location of the virtual cone is shown in figures 7-9 by dashed lines. A rough estimate of the wavelength of the undulations is computed on this cone from the approximate zero-crossings near their peak. The phase velocity $u_{p h}$ (relative to the ambient speed of sound) is then estimated from this wavelength, the indicated Strouhal number, and the known acoustic Mach number. The footprint of the second POD mode on the virtual cone demonstrates two distinct peaks for all three frequency cases. Thus, two separate phase velocities can be computed for the two peaks. The phase velocities are indicated on top of the respective sub-figures, with both the upstream and downstream values for the second POD mode appearing in that order.

Figure 10 compares the PSE modes' amplitude and phase to the unfiltered (ensemble-averaged PSD) virtual cone data, as well as the first and second line-POD modes. The most energetic Fourier modes are 
First POD mode: $u_{p h}=0.98$

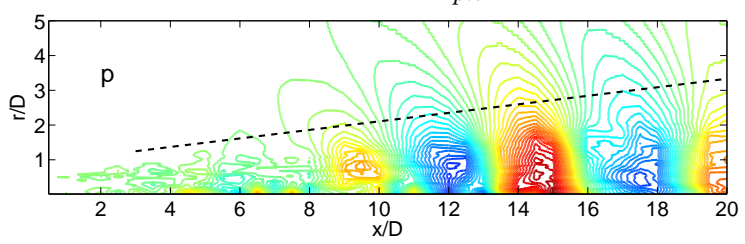

Second POD mode: $u_{p h}=0.75,0.96$

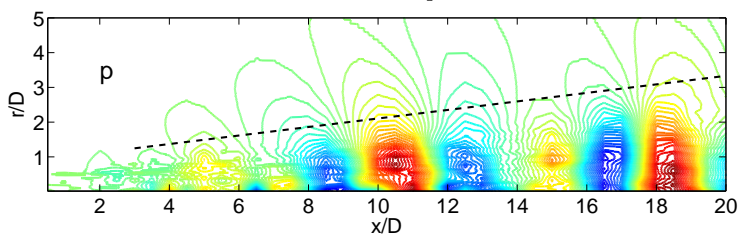

Linear PSE mode: $u_{p h}=0.83$

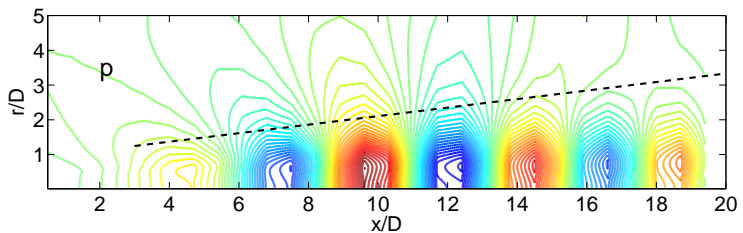

(a) $m=0$
First POD mode: $u_{p h}=0.58$

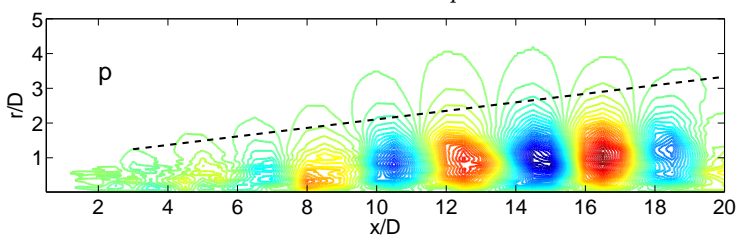

Second POD mode: $u_{p h}=0.66,0.98$

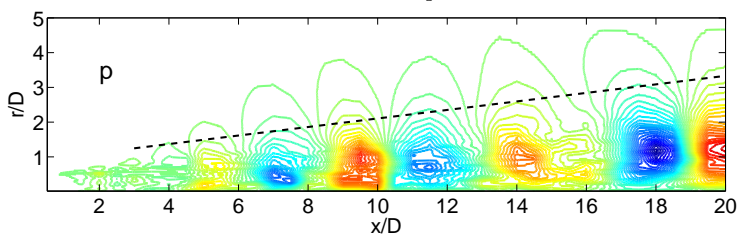

Linear PSE mode: $u_{p h}=0.73$

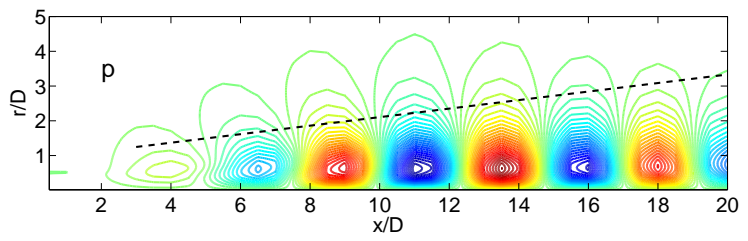

(b) $m=1$

Figure 7. Real components of pressure in the first and second planar-POD modes compared with linear PSE mode at $S t=0.1$. The dashed line represents the virtual cone.

compared here. Note that the energies of the positive azimuthal modes are not doubled to account for the corresponding negative modes. Thus, $m=0$ and $m=1$ modes have very similar energies, whereas the $m=2$ modes go down by about a factor of four in energy. As mentioned earlier, the linear PSE modes are arbitrary with respect to the amplitude and phase, and are thus rescaled and phase-shifted a posteriori.

For the subsonic jets studied by Gudmundsson \& Colonius, ${ }^{14}$ the first POD mode of the cone pressure data typically matched the PSE mode very closely. However, for some Fourier modes in heated transonic jets, the PSE mode showed greater similarity with the second POD mode. ${ }^{38}$ It was concluded thereof that as the acoustic part of the pressure field increases in dominance over the hydrodynamic, POD becomes less effective in cleanly filtering a unique wavepacket from the total fluctuations, in agreement with the acoustic radiation patterns observed in the planar-POD modes above.

In the supersonic jet here, individual POD modes also appear to be an incomplete match for the corresponding PSE modes for most Fourier modes. The exceptions appear to be the lower frequencies and higher azimuthal modes - viz. the $(S t, m)$ mode pairs of $(0.1,1),(0.1,2),(0.2,1),(0.2,2)$ and $(0.3,2)$. For the remaining Fourier modes, the PSE modes' amplitude envelopes resemble the upstream peaks of the corresponding second POD modes. The orthogonality constraint on the set of POD modes results in the near-null amplitude of the second POD mode where the first one reaches its maximum. As a consequence, the second POD modes typically display two peaks. The phase plots demonstrate that the phase speeds (the reciprocal of the slopes) of the POD modes match those of the PSE modes at upstream positions. However, the downstream peaks in the second POD modes are typically associated with acoustic radiation (as shown in figures 7-9), which is not replicated by the PSE modes. Higher phase speeds indicate acoustic instead of convective propagation of oscillations, ${ }^{14}$ reinforcing the suggested notion of dominance of the pressure fluctuations of acoustic nature over those associated with hydrodynamics downstream of the potential core closure.

It should be remarked here that the final objective of the present methodology is to employ the PSE modes to model the noise sources for a subsequent acoustic projection method to predict the noise emitted to the far field. In our previous work, a Kirchhoff surface method overlapped with the near-field microphone array - or the virtual cone - was used. Therefore, the virtual cone is the location most adequate to perform quantitative comparisons of the PSE wavepackets with the POD modes. The projection coefficients $\beta_{j}$ computed in a 
First POD mode: $u_{p h}=1.12$

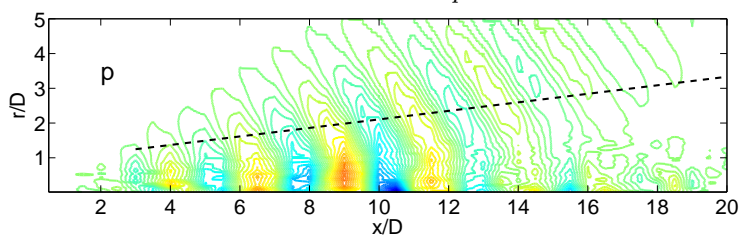

Second POD mode: $u_{p h}=1.02,1.25$

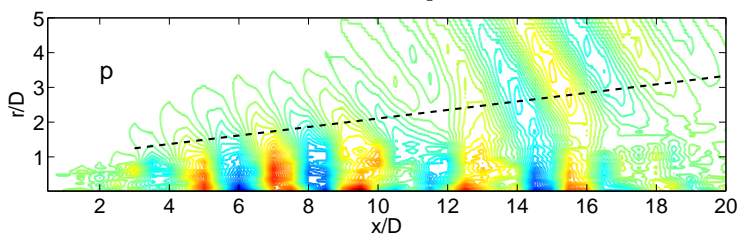

Linear PSE mode: $u_{p h}=0.95$

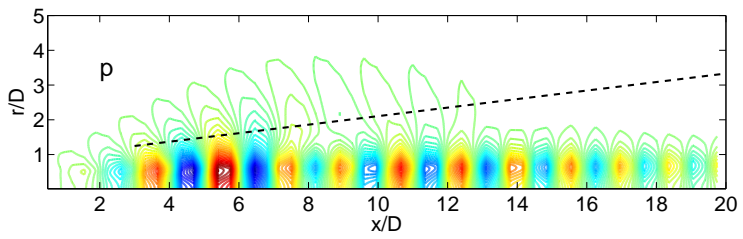

(a) $m=0$
First POD mode: $u_{p h}=0.99$

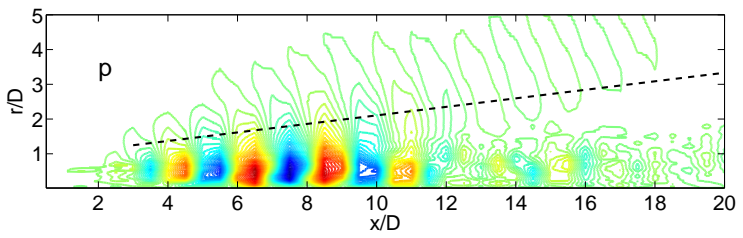

Second POD mode: $u_{p h}=1.01,1.22$

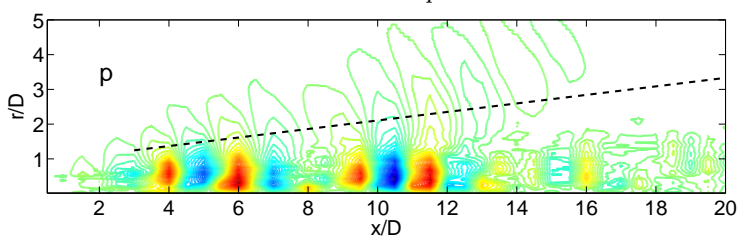

Linear PSE mode: $u_{p h}=0.94$

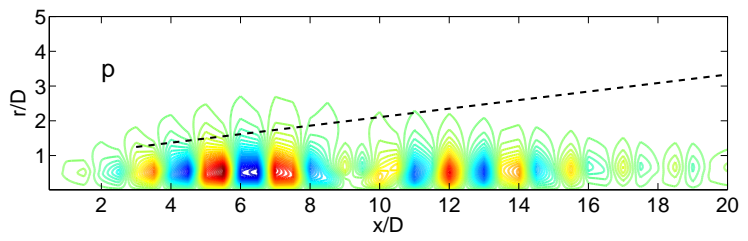

(b) $m=1$

Figure 8. Real components of pressure in the first and second planar-POD modes compared with linear PSE mode at $S t=0.3$.

similar fashion to (14) but restricting the quadrature to the cone are shown in figure 11. Compared with the projection on planar-POD modes (figure 6), very few POD modes are required to reconstruct the PSE modes, and dominance of the first and second modes is clear for most of the frequencies and azimuthal wavenumbers. The problem associated with coarse extraction of the LES data to compute the planar-POD results noted above is found here for $S t=0.5$ modes.

\section{Conclusions}

Parabolized stability equations (PSE) have been proposed as a tool for developing reduced-order models of the wavepackets implicated in peak sound radiation to the far field for turbulent jets. Previously, we have shown that linear PSE is indeed effective in predicting the near-field hydrodynamic pressure signature of wavepackets in turbulent subsonic jets. These signatures were educed from the experimental data measured at the NASA SHJAR facility by filtering with proper orthogonal decomposition (POD). The very good agreement found between the pressure imprint of linear PSE modes at the location of a conical microphone array with the POD-filtered data suggests that the main nonlinearities existing in turbulent jet flow are those between the mean flow and the small-scale random turbulence, as suggested by Mankbadi \& Liu, ${ }^{9}$ and are already accounted for in taking the turbulent mean flow as the base for the models. Present results show that, with some caveats, this is also appears to be the case for supersonic turbulent jets.

The present work focuses on a Mach 1.5 cold jet and uses LES data to validate the PSE approach. In applying these techniques to supersonic flow, there are two significant challenges. The first challenge is the existence of additional unstable waves apart from the Kelvin-Helmholtz mode that is the sole instability in subsonic jets. This new family of eigenmodes corresponds to pressure waves that resonate inside of the potential core: its members propagate downstream and upstream with respect to the jet stream forming a Mach wave system within the potential core. Similar instabilities have been found in the scope of a global eigenmode analysis. ${ }^{21}$ Computing PSE modes corresponding to this kind of instability was found to be, at least, problematic. On one hand, the upstream propagating components are necessarily damped by the parabolic marching scheme. On the other hand, the dominant Kelvin-Helmholtz instability would overwhelm the unstable, downstream propagating pressure modes and the PSE approach would converge to the Kelvin- 
First POD mode: $u_{p h}=1.50$

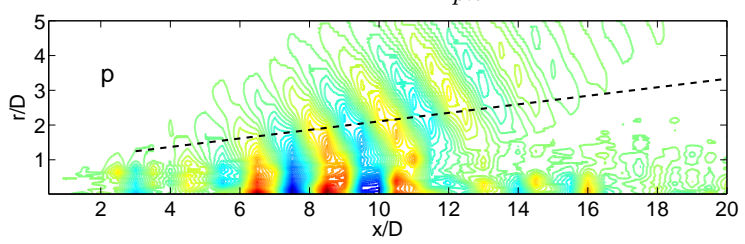

Second POD mode: $u_{p h}=1.35,1.50$

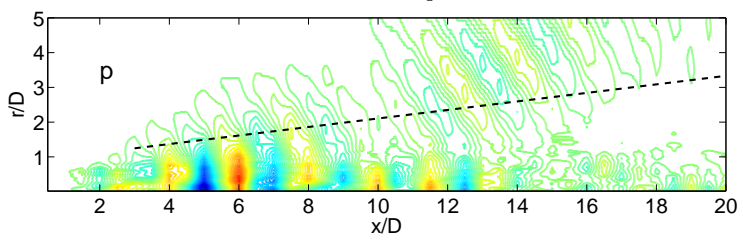

Linear PSE mode: $u_{p h}=0.98$

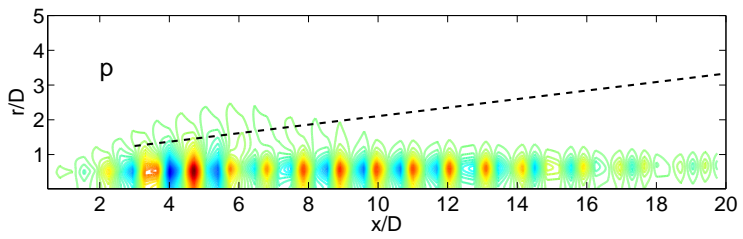

(a) $m=0$
First POD mode: $u_{p h}=1.43$

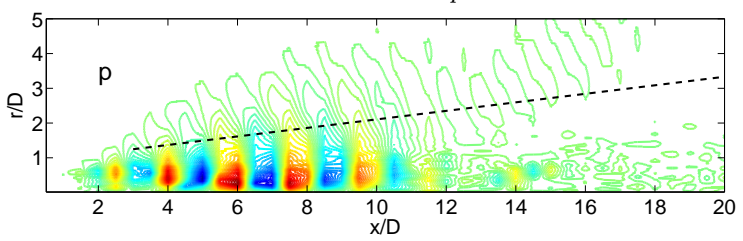

Second POD mode: $u_{p h}=0.64,1.46$

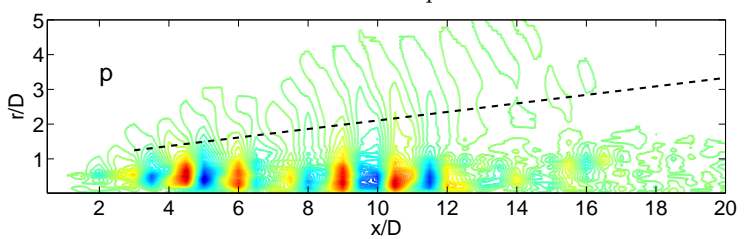

Linear PSE mode: $u_{p h}=0.91$

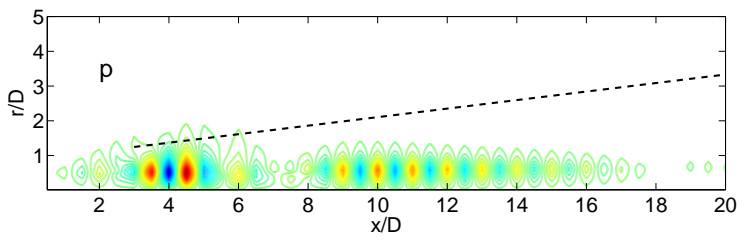

(b) $m=1$

Figure 9. Real components of pressure in the first and second planar-POD modes compared with linear PSE mode at $S t=0.5$.

Helmholtz mode alone. A study of the relative contributions of the different instability modes to the total fluctuations at the inlet of the PSE integration domain was performed using the bi-orthogonal eigenfunction system delivered by linear stability theory. It was found that the fluctuation's amplitude corresponding to the Kelvin-Helmholtz eigenmode was much higher that those associated with other instability waves, so that most of the fluctuation kinetic energy was contained in this mode alone. The conclusion is that for this particular jet configuration, the Kelvin-Helmholtz instability dominates over the core pressure waves, and the use of PSE as model for the large-scale hydrodynamic structures is justified.

The other challenge posed by supersonic jets is the increased acoustic content of the near field, that hinders the extraction of the PSE-modeled hydrodynamic part. Compared to earlier experimental data, the LES provides greater wealth of information. One novel possibility is a POD on the full pressure field (instead of restricting attention to the near-field microphone 'cage' used at NASA SHJAR or UTRC), and this is pursued for the validation of the linear PSE model. The POD eigenspectra are relatively flat for most Fourier modes, indicating that no single mode can be expected to retrieve the PSE solution from the unfiltered fluctuations. However, it is shown here that relatively few POD modes (1 to 5) are required to reconstruct the PSE mode at frequencies up to $S t=0.4$, thereby lending confidence to our modeling technique. At higher frequencies, the resolution on the interpolation grid (used to reduce the LES data and make it tractable) proved insufficient to avoid aliasing. The POD modes also display significant acoustic propagating to the aft direction, that cannot be captured by the PSE for the reasons explained above. In fact, for the $S t=0.3, m=0$ mode, which is close to the most efficient radiation condition, the first 5 POD modes were required for reconstructing the PSE solution. The conclusion can be drawn that POD may not be optimal for extracting the hydrodynamic signatures of the wavepackets that PSE is attempting to model, especially as the relative importance of acoustic radiation increases with jet temperature and Mach number. However, whenever the contributions from hydrodynamic and acoustic can be discerned, good agreement between the linear PSE model and the hydrodynamic component is found.

This work was supported by NAVAIR through an STTR grant N68335-11-C-0026 to Cascade Technologies, Inc. and by ONR Grant N00014-11-1-0753. The authors would like to thank Dr. Joseph W. Nichols and Prof. Sanjiva K. Lele, of Stanford University, for their contributions to the LES efforts and their inputs on global modes. The LES calculations were carried out on CRAY XE6 machines at DoD supercomputer 

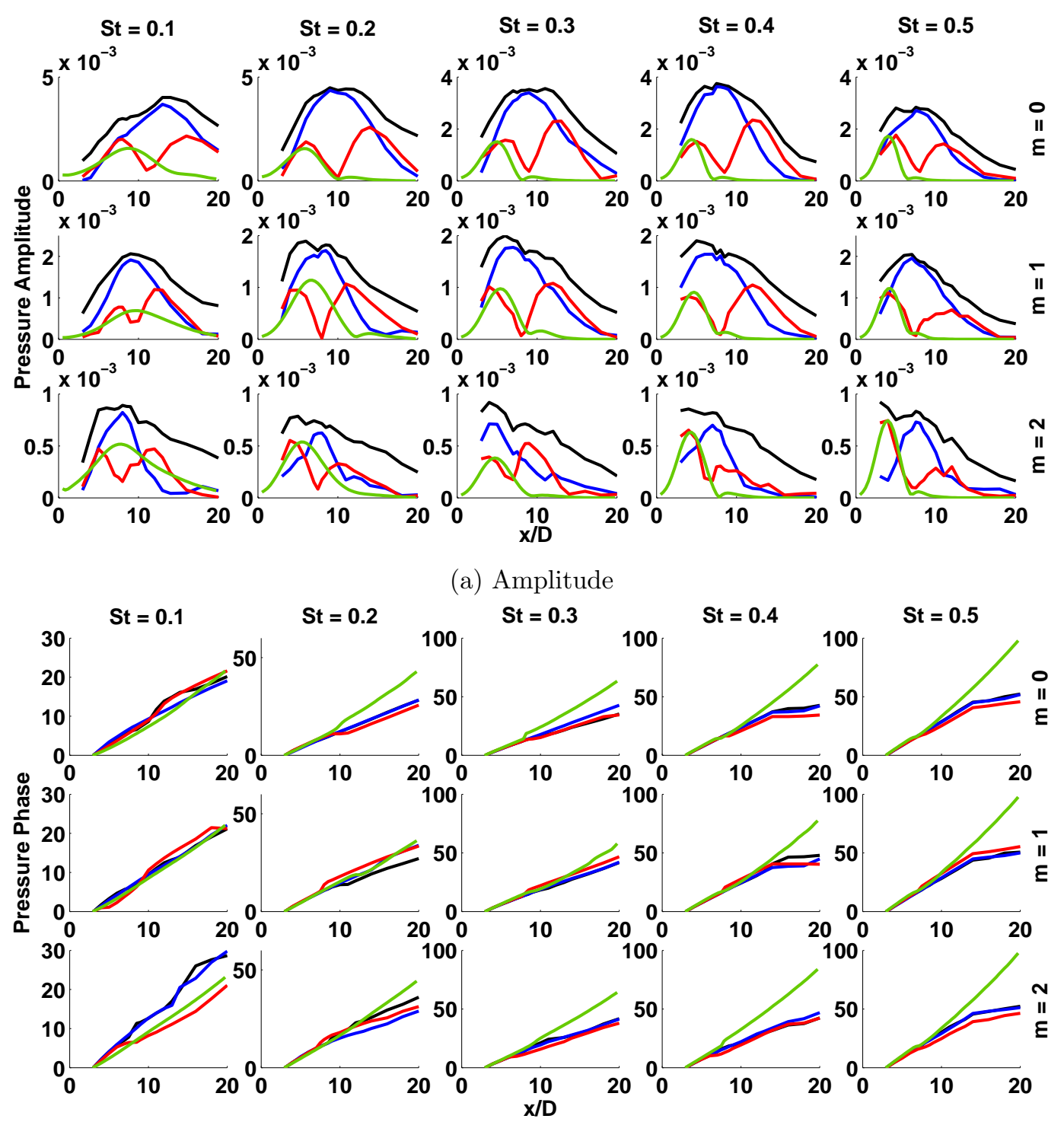

(b) Phase

Figure 10. (a) Pressure amplitudes and (b) phases extracted at the location of the virtual cone, as computed by solution of linear PSE, compared with data extracted from the LES database. Green: linear PSE. Black: unfiltered pressure from LES. Blue: 1st line-POD mode. Red: 2nd line-POD mode.

facilities in ERDC and AFRL.

A. Appendix: Linear operators in the PSE formulation

$$
\mathcal{L}^{0}=\left(\begin{array}{ccccc}
\partial_{x} \bar{u}_{x} & \partial_{r} \bar{u}_{x} & 0 & 0 & 0 \\
\partial_{x} \bar{u}_{r} & \partial_{r} \bar{u}_{r} & 0 & 0 & 0 \\
0 & 0 & \bar{u}_{r} / r & 0 & 0 \\
0 & 0 & 1 / r & \gamma \bar{\Theta} & 0 \\
\partial_{x} \bar{\zeta} & \partial_{r} \bar{\zeta}-\bar{\zeta} / r & 0 & 0 & -\bar{\Theta}
\end{array}\right) \quad \mathcal{L}^{t}=\left(\begin{array}{ccccc}
1 & 0 & 0 & 0 & 0 \\
0 & 1 & 0 & 0 & 0 \\
0 & 0 & 1 & 0 & 0 \\
0 & 0 & 0 & 0 & 0 \\
0 & 0 & 0 & 0 & 1
\end{array}\right)
$$




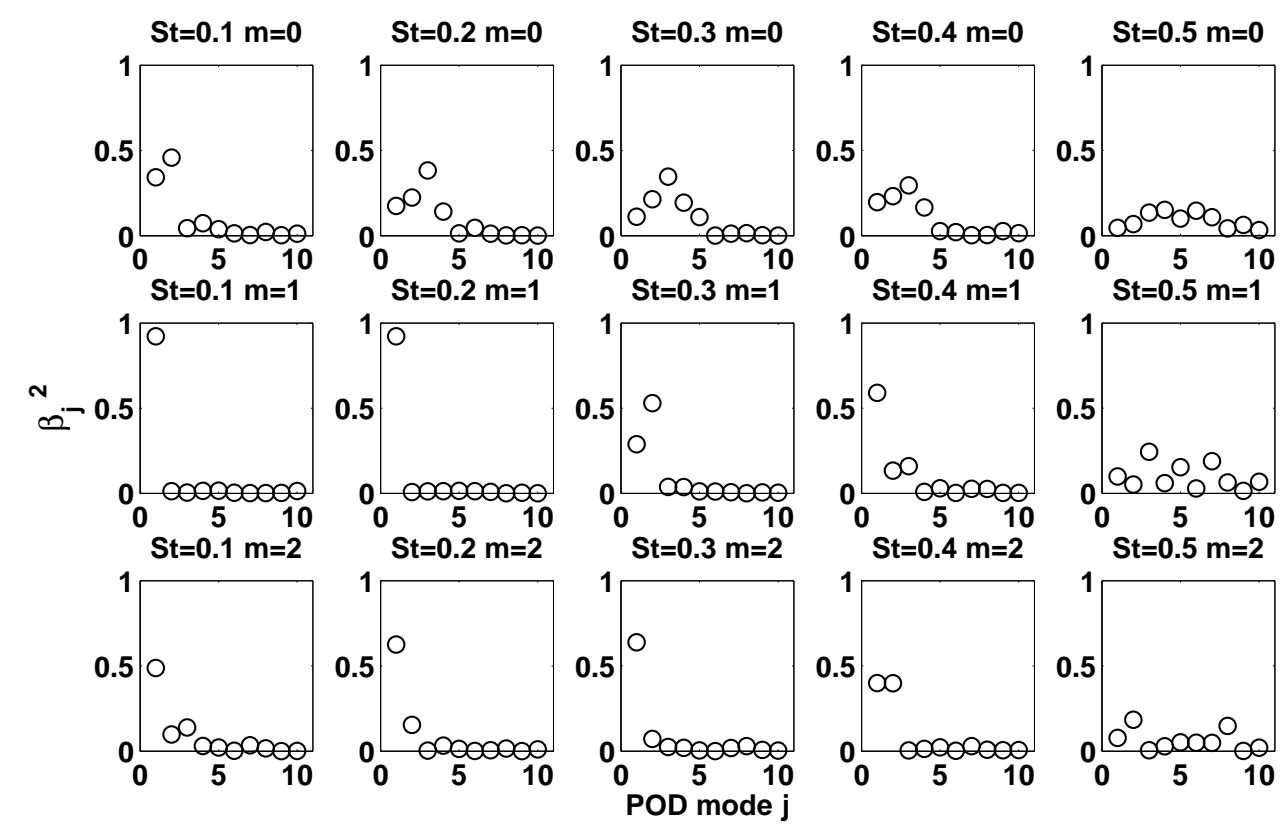

Figure 11. Squared magnitudes of projection coefficients $\beta_{j}$ for the respective PSE solutions on to the line-POD modes.

$$
\begin{aligned}
& \mathcal{L}^{x}=\left(\begin{array}{ccccc}
\bar{u}_{x} & 0 & 0 & 0 & \bar{\zeta} \\
0 & \bar{u}_{x} & 0 & 0 & 0 \\
0 & 0 & \bar{u}_{x} & 0 & 0 \\
1 & 0 & 0 & 0 & \bar{u}_{x} \\
-\bar{\zeta} & 0 & 0 & \bar{u}_{x} & 0
\end{array}\right) \quad \mathcal{L}^{\theta}=\left(\begin{array}{ccccc}
0 & 0 & 0 & 0 & 0 \\
0 & 0 & 0 & 0 & 0 \\
0 & 0 & 0 & 0 & \bar{\zeta} \\
0 & 0 & -\bar{\zeta} & 0 & 0 \\
0 & 0 & 1 & 0 & 0
\end{array}\right) \\
& \mathcal{V}^{0}=\left(\begin{array}{ccccc}
0 & 0 & 0 & 0 & -\nabla^{2} \bar{u}_{x}-(1+\lambda) \frac{\partial \bar{\Theta}}{\partial x} \\
0 & \frac{(2+\lambda) \bar{\zeta}}{r^{2}} & 0 & 0 & -\nabla^{2} \bar{u}_{r}+\frac{\bar{u}_{r}}{r^{2}}-(1+\lambda) \frac{\partial \bar{\Theta}}{\partial r} \\
0 & 0 & \frac{\bar{\zeta}}{r^{2}} & 0 & 0 \\
0 & -(\gamma-1)\left(4 \frac{\bar{u}_{r}}{r^{2}}+\frac{2 \lambda \bar{\Theta}}{r}\right) & 0 & -\frac{\gamma \nabla^{2} \bar{\zeta}}{P r_{\infty}} & \\
0 & 0 & 0 & 0 & 0
\end{array}\right) \\
& \mathcal{V}^{x}=\left(\begin{array}{ccccc}
0 & -\frac{(1+\lambda) \bar{\zeta}}{r} & 0 & 0 & 0 \\
0 & 0 & 0 & 0 & 0 \\
0 & 0 & 0 & 0 & 0 \\
0 & 0 & 0 & -(\gamma-1)\left(4 \partial_{x} \bar{u}_{x}+2 \lambda \bar{\Theta}\right) & -2(\gamma-1)\left(\partial_{x} \bar{u}_{r}+\partial_{r} \bar{u}_{x}\right) \\
0 & 0 & 0 & 0 & 0
\end{array}\right) \\
& \mathcal{V}^{r}=\left(\begin{array}{ccccc}
-\bar{\zeta} / r & 0 & 0 & 0 & 0 \\
0 & \frac{(2+\lambda) \bar{\zeta}}{r^{2}} & 0 & 0 & \bar{\zeta} \\
0 & 0 & -\bar{\zeta} / r & 0 & 0 \\
-2(\gamma-1)\left(\partial_{x} \bar{u}_{r}+\partial_{r} \bar{u}_{x}\right) & -(\gamma-1)\left(4 \partial_{r} \bar{u}_{r}+2 \lambda \bar{\Theta}\right) & 0 & -\gamma \bar{\zeta} /\left(r P r_{\infty}\right) & -1 /\left(r P r_{\infty}\right) \\
0 & 0 & 0 & 0 & 0
\end{array}\right) \\
& \mathcal{V}^{\theta}=\left(\begin{array}{ccccc}
0 & 0 & 0 & 0 & 0 \\
0 & 0 & \frac{(3+\lambda) \bar{\zeta}}{r} & 0 & 0 \\
0 & -\frac{(3+\lambda) \bar{\zeta}}{r} & 0 & 0 & 0 \\
0 & 0 & -(\gamma-1)\left(4 \frac{\bar{u}_{r}}{r}+2 \lambda \bar{\Theta}\right) & 0 & 0 \\
0 & 0 & 0 & 0 & 0
\end{array}\right)
\end{aligned}
$$




$$
\begin{aligned}
& \mathcal{V}^{x x}=\left(\begin{array}{ccccc}
-(2+\lambda) \bar{\zeta} & 0 & 0 & 0 & 0 \\
0 & -\bar{\zeta} & 0 & 0 & 0 \\
0 & 0 & -\bar{\zeta} & 0 & 0 \\
0 & 0 & 0 & -\frac{\gamma \bar{\zeta}}{P r_{\infty}} & -\frac{1}{P r_{\infty}} \\
0 & 0 & 0 & 0 & 0
\end{array}\right) \quad \mathcal{V}^{r r}=\left(\begin{array}{ccccc}
-\bar{\zeta} & 0 & 0 & 0 & 0 \\
0 & -(2+\lambda) \bar{\zeta} & 0 & 0 & 0 \\
0 & 0 & -\bar{\zeta} & 0 & 0 \\
0 & 0 & 0 & -\frac{\gamma \bar{\zeta}}{P r_{\infty}} & -\frac{1}{P r_{\infty}} \\
0 & 0 & 0 & 0 & 0
\end{array}\right)
\end{aligned}
$$

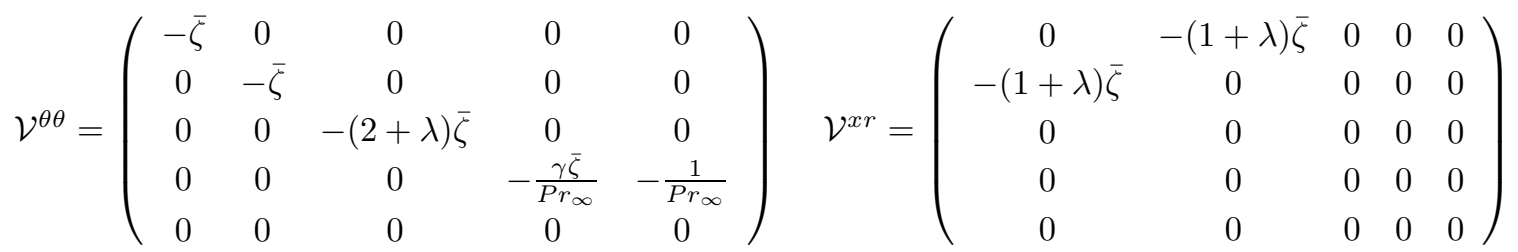

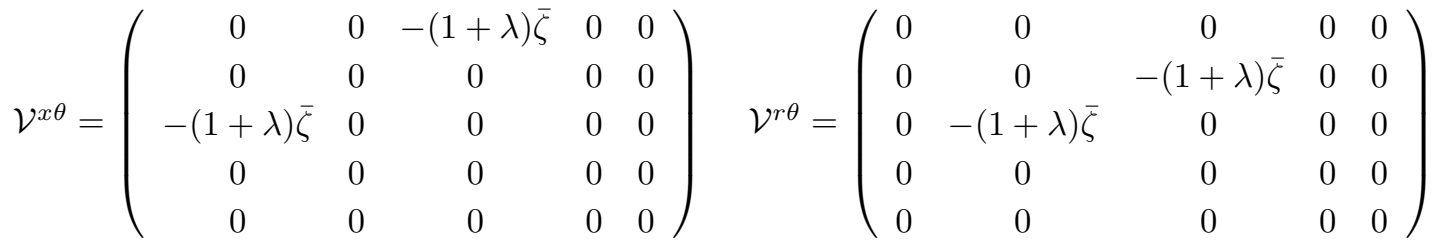

where $\bar{\Theta}=\partial_{x} \bar{u}_{x}+\partial_{r} \bar{u}_{r}+\bar{u}_{r} / r$.

\section{References}

${ }^{1}$ C. Tam, F. Hu, On the three families of instability waves of high-speed jets, J. Fluid Mech. 201 (1989) $447-483$.

${ }^{2}$ Y. Khalighi, J. W. Nichols, F. Ham, S. K. Lele, P. Moin, Unstructured Large Eddy Simulation for Prediction of Noise Issued from Turbulent Jets in Various Configurations, AIAA Paper 2011-2886.

${ }^{3}$ C. Tam, D. Burton, Sound generated by instability waves of supersonic flows. Part 2. Axisymmetric jets., J. Fluid Mech. 138 (1984) 273-295.

${ }^{4}$ E. Mollo-Christensen, Jet noise and shear flow instability seen from an experimenter's viewpoint, J. Applied Mech. 34 (1967) $1-7$

${ }^{5}$ A. Hussain, W. Reynolds, The mechanics of an organized wave in turbulent shear flow, J. Fluid Mech. 42 (1970) $241-$ 258.

${ }^{6}$ W. Reynolds, A. Hussain, The mechanics of an organized wave in turbulent shear flow. Part 3. Theoretical models and comparisons with experiments, J. Fluid Mech. 54 (1972) 263 - 288.

${ }^{7}$ M. Gaster, E. Kit, I. Wygnanski, Large-scale structures in a forced turbulent mixing layer, J. Fluid Mech. 150 (1985) 23-39.

${ }^{8}$ B. Marasli, F. H. Champagne, I. Wygnanski, Modal decomposition of velocity signals in a plane, turbulent wake, J. Fluid Mech. 198 (1989) 255-273.

${ }^{9}$ R. Mankbadi, J. T. C. Liu, A study of the interactions between large-scale coherent structures and fine-grained turbulence in a round jet, Proc. Roy. Soc. London 1443 (1981) 541-602.

${ }^{10}$ J. T. C. Liu, Developing large-scale wavelike eddies and the near jet noise field, J. Fluid Mech. 62 (1974) 437-464.

${ }^{11}$ P. Morris, M. Giridharan, G. Lilley, On the turbulent mixing of compressible free shear layers, Proc. R. Soc. Lond. A. 431 (1990) 219-243.

${ }^{12}$ C. Tam, P. Morris, Tone excited jets - Part V: A theoretical model and comparison with experiments, J. Sound Vib. 102 (1985) 119-151.

${ }^{13}$ T. Suzuki, T. Colonius, Instability waves in a subsonic round jet detected using a near-field phased microphone array, J. Fluid Mech. 565 (2006) 197-226.

${ }^{14} \mathrm{~K}$. Gudmundsson, T. Colonius, Instability wave models for the near-field fluctuations of turbulent jets, J. Fluid Mech. 689 (2011) 97-128.

${ }^{15}$ F. P. Bertolotti, T. Herbert, Analysis of the linear stability of compressible boundary layers using the PSE, Theoret. Comput. Fluid Dyn. 3 (1991) 117-124.

${ }^{16}$ F. P. Bertolotti, T. Herbert, P. Spalart, Linear and nonlinear stability of the blasius boundary layer, J. Fluid Mech. 242 (1992) 441-474.

${ }^{17}$ T. Herbert, Parabolized stability equations, Annu. Rev. Fuid Mech. 29 (1997) 245-283.

${ }^{18}$ D. Rodriguez, A. Samanta, A. Cavalieri, T. Colonius, P. Jordan, Parabolized Stability Equation models for predicting large-scale mixing noise of turbulent round jets, AIAA Paper 2011-2838.

${ }^{19}$ N. Sandham, A. Salgado, Nonlinear interaction model of subsonic jet noise, Phil. Trans. Roy. Soc. A 366 (2008) 2745-2760.

${ }^{20}$ M. R. Malik, C. L. Chang, Nonparallel and nonlinear stability of supersonic jet flow, Computers and Fluids 29 (2000) $327-365$.

${ }^{21}$ J. W. Nichols, S. K. Lele, Global modes and transient response of a cold supersonic jet, J. Fluid Mech. 669 (2011) $225-241$.

${ }^{22}$ J. Bridges, A. Hussain, Roles of initial condition and vortex pairing in jet noise, J. Sound Vib. 2 (117) (1987) $289-311$. 
${ }^{23}$ K. B. M. Q. Zaman, Effect of nozzle exit conditions on subsonic jet noise, AIAA Paper 2011-2704.

${ }^{24} \mathrm{H}$. Salwen, C. Grosch, The continuous spectrum of the orr-sommerfeld equation. Part II. Eigenfunction expansions, J. Fluid Mech. 104 (1981) 445-465.

${ }^{25}$ D. Hill, Adjoint systems and their role in the receptivity problem for boundary layers, J. Fluid Mech. 292 (1995) $183-204$.

${ }^{26}$ A. Tumin, Three-dimensional spatial normal modes in compressible boundary layers, J. Fluid Mech. 586 (2007) $295-322$.

${ }^{27}$ G. A. Brès, J. W. Nichols, S. K. Lele, F. E. Ham, R. H. Schlinker, R. A. Reba, J. Simonich, Unstructured large eddy simulation of a hot supersonic over-expanded jet with chevrons, 18th AIAA/CEAS Aeroacoustics Conference and Exhibit, 4-6 June, AIAA, Colorado Springs, CO, 2012.

${ }^{28}$ G. A. Brès, J. W. Nichols, S. K. Lele, F. E. Ham, Towards best practices for jet noise predictions with unstructured large eddy simulations, 42nd AIAA Fluid Dynamics Conference and Exhibit, June 25-28, AIAA, New Orleans, LA., 2012.

${ }^{29}$ P. Holmes, J. Lumley, G. Berkooz, Turbulence, Coherent structures, dynamical systems and symmetry, Cambridge University Press, New York, 1996.

${ }^{30}$ J. B. Freund, T. Colonius, POD analysis of sound generation by a turbulent jet, AIAA Paper 2002-0072.

${ }^{31}$ L. Sirovich, Chaotic dynamics of coherent structures. parts i-iii, Quarterly of Applied Math. XLV (3) (1987) $561-582$.

${ }^{32}$ R. Schlinker, R. Reba, J. Simonich, T. Colonius, K. Gudmundsson, F. Ladeinde, Towards prediction and control of large-scale turbulent structure supersonic jet noise, Proceedings of ASME Turbo Expo 2009 (GT2009-60301).

${ }^{33} \mathrm{~K}$. Thompson, Time dependent boundary conditions for hyperbolic systems, J. Comp. Phys. 68 (1987) 1-24.

${ }^{34}$ K. Mohseni, T. Colonius, Numerical treatment of polar coordinate singularities, J. Comp. Phys. 157 (2000) $787-795$.

${ }^{35}$ F. Li, M. R. Malik, Spectral analysis of parabolized stability equations, Comp. Fluids 26 (3) (1997) $279-297$.

${ }^{36}$ A. Tumin, M. Amitay, J. Cohen, M. Zhou, A normal multimode decomposition method for stability experiments, Phys. Fluids 8 (10) (1996) 2777-2779.

${ }^{37}$ A. Tumin, Multimode decomposition of spatially growing perturbations in a two-dimensional boundary layer, Phys. Fluids 15 (2003) 2525-2540.

${ }^{38} \mathrm{~K}$. Gudmundsson, Instability wave models of turbulent jets from round and serrated nozzles, Ph.D. thesis, California Institute of Technology (2010). 